Chapter 8

\title{
Human Pluripotent Stem Cell Applications in Drug Discovery and Toxicology - An overview
}

\author{
Shiva Prasad Potta, Tomo Šarić, Michael Heke, \\ Harinath Bahudhanapati and Jürgen Hescheler \\ Additional information is available at the end of the chapter \\ http://dx.doi.org/10.5772/58485
}

\section{Introduction}

Various drugs are being introduced into market for generating beneficial therapeutic effects in humans. The pharmaceutical industry invests about $\$ 1.5$ billion over the time period of $10-15$ years to take a candidate drug from primary screen to market. Unfortunately, many drugs are withdrawn due to side effects associated with off-and on-target toxicity [1]. For example, as many as nine out of ten promising candidates beginning clinical phase I will not achieve marketing approval [2] and only $20 \%$ of agents that show efficacy against cardiovascular diseases in preclinical development are licensed after demonstrating sufficient efficacy in phase III testing [3]. The success rate in anticancer drug development process is with $5 \%$ of licensed agents even lower. Off-target cardiac toxicity is the most common cause of regulatory delay in approval and market withdrawal of newly developed pharmaceuticals $[4,5]$. Druginduced sudden cardiac death and ventricular arrhythmia caused the withdrawal of more drugs in recent years than any other adverse drug reaction. Moreover, over 100 non-cardiac drugs are suspected to be of high-risk and carry cardiovascular-related black box warnings [6]. Similar considerations are raised concerning arrhythmia and toxicity induced by environmental factors, including industrial chemicals, food additives, cosmetics, and others, as outlined in the European REACH initiative (Registration, Evaluation, Authorization and Restriction of Chemical substances).

Current drug safety evaluation processes that are required for regulatory purposes mostly rely on animal studies and immortalized cell-based assays due to lack of suitable human in vitro cell systems. In Europe, almost 10 million vertebrate animals are used annually for research. Although highly predictive assays involving whole heart or slice preparations and in vivo animal testing remain the standard for preclinical safety pharmacology, this extensive use of 
animals and their tissues does not eliminate high attrition rates of novel drugs. One of the major reasons for this is limited predictability of existing preclinical animal (and cellular) models for assessment of drug safety and efficacy. Animal models do not always predict the toxicity in humans with sufficient accuracy because of inter-species differences [7]. For example, murine and human hearts greatly differ in some aspects of electrophysiological properties [8]. In addition, inbred animals that are frequently used in these analyses do not mimic the genetic diversity of human population required for accurate prediction of drug responses [9]. Therefore, identification of reliable and robust human cell systems for toxicity assessment has become a driving interest for pharmaceutical industries.

In cardiac area different types of tests are already playing an important role in reducing costs and drug attrition rates. These strategies involve a tiered system which starts with in vitro single cell analyses followed by tests with ex vivo tissues and organs and progresses to in vivo animal models and, finally, clinical trials [10]. The most important in vitro test consists of automated patch-clamp recordings of Chinese hamster ovary $(\mathrm{CHO})$ cells expressing human Ether-à-go-go-Related Gene (hERG) channel. This test is being used to identify compounds that block hERG channel and prolong cardiac action potential (AP) duration (i.e. the QT interval) predisposing to Torsade de pointes tachycardia and sudden cardiac death [11]. The assessment of the torsadogenic potential of each compound in the drug discovery process also includes determination of drug's ability to prolong the AP in isolated, arterially perfused rabbit ventricular wedge preparations or canine Purkinje fibers and monitoring of heart rates and occurrence of arrhythmia in animals. Each of these endpoints has it's own specificity and sensitivity [12]. For example, hERG-expressing $\mathrm{CHO}$ cells lack the complexity of native CMs and cannot accurately predict the organ toxicity or lethal and arrhythmogenic side effects of compounds that block other channels or signaling pathways. Therefore, additional in vitro assays that better recapitulate human pathophysiology and diversity are needed to better predict all potential on-and off-target toxicities, reduce drug attrition rates and avoid use of animals for testing drugs that would never reach clinical application.

Pluripotent stem cells (PSCs) have unrestricted proliferation capacity, are able to differentiate into any differentiated cell type thus offering a cost-effective unlimited and invaluable source of organotypic differentiated cells relevant to assess human long-term organ toxicity. The ethical issues associated with human embryonic stem cells (ESCs) were a major concern in their application in toxicity studies. However, the Nobel prize-winning discovery that transient expression of a few transcription factors can stably convert an adult somatic cell into an early embryonic stage, i.e. into so called induced pluripotent stem cells (iPSCs) [13], has opened new possibilities in drug discovery circumventing ethical issues and problematic accessibility.

Repeated dose toxicity (RDT) occurs after repeated exposure to a substance over certain period of time. In the context of cosmetics, which are generally used for months and years, long-term RDT testing is of particular importance and forms the integral part of the quantitative risk assessment. The prediction of endpoints and hazard identification of both newly developed and existing cosmetic ingredients in humans is mainly based on the animal systems as they allow simultaneous evaluation of multiple organ systems. However, there is a great demand 
and need for the development of multidisciplinary integrated approaches consisting of human in vitro models for risk assessment as an alternative to animal models as they better mimic the human in vivo system [14].

In this chapter, we will summarize the latest developments in applications of PSCs and their tissue-specific derivatives for toxicity testing. We will outline the recent developments in toxicogenomic technologies which are employed to develop and investigate human biomarkers for toxicity in PSC based models accelerating drug development process. We mainly focus on the application of PSCs in RDT testing.

\section{Human pluripotent stem cells for repeated dose toxicity assessment}

Human pluripotent stem cells (PSC) offer with their ability to recapitulate the most essential steps of embryonic development and give rise to different mature cell types in vitro an optimal human cellular model, which could help in increasing the safety and predictability of RDT testing leading to low late stage attrition of compounds. Combined with this cell model, toxicogenomic technologies would help predict biomarkers in an evidence-based approach.

So far, the safety assessment for novel drug candidates includes in vivo RDT tests in rodent and non-rodent models. The drawbacks of RDT studies include false negative results and unexpected humans toxicity of compounds that were judged to be safe in preclinical studies $[15,16]$. Such unexpected toxicity is one of the major reasons for the withdrawal of a drug from the market. The heart and liver are often target organs in toxicology. Novel in vitro screening methods are, thus, required to classify toxic compounds earlier in development, which would lead to safer drugs, more efficient drug discovery process, lower costs and reduced laboratory animal use [17]. There is an increasing interest from biopharmaceutical industry to develop such test systems by using derivatives of human ESCs or iPSCs.

The iPSCs have a clear advantage over ESCs as they do not involve ethical issues. The generation of iPSCs involves reorganization of condensed chromatin to open state chromatin, which is aided by histone acetylation. Epigenetic factors are crucial for iPSC generation and maintenance of their pluripotent state. Although the epigenetic state of iPSCs largely resembles that of ESCs, iPSCs also have a unique DNA methylation patterns they retain epigenetic memory of the respective somatic tissue of origin which might influence their differentiation potential and affect the quality and quantity of cells for RDT [18]. On the other side, it is also well known that different agents, so called epimutagens, can cause DNA methylation and histone modification changes leading to disease [19]. These epigenetic modifications directly affect transcription factors and other chromatin binding proteins that regulate cell type-specific gene expression. The detection of biomarkers related to epigenetic modifications in RDT would be of great importance, but until now there are no systematic studies conducted in this direction. In addition, employing of iPSCs and their derivatives for this purpose poses a great challenge because genetic and epigenetic variations in iPSCs associated with reprogramming and in vitro manipulation may compromise their utility for downstream applications [20]. The lesser the variation in epigenetic changes in iPSCs the greater will be the specificity in in vitro 
toxicological studies. Recently, Planello and coworkers demonstrated that the choice of reprogramming factors greatly influences the DNA methylation abnormalities in iPSCs. Even highly selected iPSC lines have been shown to retain epigenetic signature of donor cell [21]. Gupta et al have shown that global transcriptional profiles of human iPSCs and ESCs are very similar and that this similarity also exists between the corresponding beating clusters derived from them [22]. They have also shown that some fibroblasts-specific mRNA expression partners were retained in the iPSCs derived from them. Significant proportion of these genes were also shown to be expressed at the same level in iPSC-derived but not in ESC-derived beating clusters indicating the retention of epigenetic memory even in the differentiated and highly enriched iPSC derivatives. Likewise, several microRNA expression profiling studies have shown the subtle differences between iPSC derivatives [23]. Hence, the iPSCs may not represent an ideal platform for RDT testing. With the current pace of iPSC research it may be possible to create iPSCs with little or no epigenetic anomalies. Polo and coworkers have shown that this retained epigenetic memory of iPSCs in early passages can be erased using extensive continued passage [24]. By using chromatin-modifying compounds like HDAC inhibitors it may be possible to stabilize the epigenetic state of iPSCs and their derivatives and decrease the frequency of heterogeneity within iPSCs. However, using the PSC-derivatives to predict RDT in human toxicological endpoints is still challenging.

\section{PSC-derived cardiomyocytes for toxicity testing}

Human Stringent cosmetics legislation amending directives especially within the European Union (EU) related to complete replacement of animal models in cosmetic industry safety testing by alternative methods has emphasized an urgent need for the development of reduction, refinement and replacement (3R) of the existing animal studies [25]. In order to fill gaps in non-animal alternative methods and to focus on complex RDT a research initiative called "Safety Evaluation Ultimately Replacing Animal Testing 1 (SEURAT-1)" composed of six complimentary research projects was launched in 2011 and jointly funded by the European Commission's FP7 HEALTH Programme and Cosmetics Europe (http://www.seurat-1.eu/). Embryonic Stem cell-based Novel Alternative Testing Strategies (ESNATS) is a European Union's Seventh Framework Programme (FP7), which focuses on developing a human ESC based novel toxicity test platforms to accelerate drug development. Human ESC based in vitro reproductive toxicity, neurotoxicity, toxicogenomics, proteomics and kinetics were tested for their predictive value in the identification of toxicity endpoints (http://www.esnats.eu). The RDT delivers the No Observable Adverse Effect (NOAEL), which is used in calculation of the substance safety parameters [25]. PSC-derived models hold a great potential for refinement of current models of cardiotoxicity. For many toxicology applications, a homogeneous defined population of specific cell types is required which stem cells can provide.

Validity of human PSC-derived cardiomyocytes (CM) for toxicity testing and safety pharmacology has been investigated in several studies [26-28]. The susceptibility of disease-specific human iPSC-CMs to toxicity compared to healthy human PSC-CMs was evaluated recently by Joseph $\mathrm{Wu}$ laboratory [29]. This group showed that disease-specific human iPSC-CM are 
more accurate predictors of drug-induced cardiotoxicity than standard hERG-expressing HEK293 cells. This observation suggests that human iPSC-CM may represent a suitable model for evaluation of drug safety and efficacy. However, there is still a need to examine how well the alternative systems can replace the animal models for RDT testing. The traditional repeated-dose toxicological endpoints that relate to cardiotoxicity include histopathological examinations of the heart and electrocardiographic recordings in the non-rodent species [30]. The current regulatory framework guidelines for cardiotoxicity testing include blood pressure, heart rate and electrocardiogram (ECG) parameters as well as repolarization and conductance abnormalities, cardiac output, ventricular contractility and vascular resistance. The limitations of RDT testing in vivo clearly encouraged the scientific community to identify and develop alternative in vitro methodologies to thoroughly estimate the integrated and complex responses in the endpoints that are taken into consideration.

Drugs exerting toxic effects on cardiovascular system have shown to affect the heart function in a way that includes changes in the contractility, cardiac rhythm, blood pressure and ischemia [31]. Such toxic effects have led these drugs to be withdrawn, requiring expansion of rules on cardiotoxicity testing. A new application for CMs derived from human ESCs and iPSCs has surfaced because of the lack of availability of human primary material for cardiotoxicity testing and their ability to overcome species variability. In vitro cardiotoxicity testing applications using human PSC-CMs is very advantageous and complimentary to the existing RDT applications. End points such as action potential parameters, metabolic activity, membrane leakage, energy content and intracellular calcium handling can be monitored for assessing cardiotoxicity. As mentioned above, the effect of new drugs on cardiac electrophysiology (i.e. changes in ventricular repolarization) is a focus for tight control. The balanced concerted activity of several cardiac ion channels is important for proper ventricular repolarization and alterations may lead to ventricular arrhythmias. Therefore, electrophysiological assessment of the proarrhythmic potential of drugs is very relevant in cardiotoxicity assays and human PSCderived CMs are suitable for such assays because they exhibit calcium handling properties, ion channel activity and regulatory protein expression important for the development of a mature repolarization phenotype in CMs [12].

Recently, several studies evaluated the potential of human iPSC-CMs for pharmacological screening-assays and drug discovery applications [32-34]. However, the utility of iPSC-CMs to accurately predict toxicity in humans may be limited by their immature character [35]. Current differentiation protocols give rise to heterogeneous phenotypes of spontaneously beating human PSC-CMs with structural proteins, Ca2+release units, ion channels, action potentials, and hormonal response being similar to that of native fetal CMs. However, the electrophysiological and structural properties of PSC-CMs do not fully resemble those of adult CMs. Therefore, the model based on human PSC-CMs must be improved before it can represent an ideal platform for cardiac RDT. The mentioned issues can be solved by following measures: a) by modulating cellular signaling pathways it is possible to get a homogeneous $\mathrm{CM}$ population [36] b) with the application of tissue engineering it is possible to create a $3 \mathrm{D}$ tissue constructs which provide microenvironment similar to native heart thus helping in structural maturation of CMs [37] and c) prolonged culturing of iPSC-CMs can increase the 
maturation of Ca2+handling [38]. Further improvements of differentiation methods will enable generation of more homogeneous and mature $\mathrm{CM}$ populations thus increasing their validity for RDT testing. The overall predictability of drug efficacy and toxicity using iPSC-CMs and disease specific iPSC-CMs has been recently reported by several groups [29, 39-41]. An absolute requirement for $\mathrm{CMs}$ to be used for RDT is to be able to stably maintain the beating cardiac phenotype for a prolonged period of time under defined conditions. Both hiPSC-CMs and hESC-CMs display beat rate variability similar to that of a human heart sinoatrial node [42]. However, recently, variability in action potentials and sodium currents in response to lidocaine and tetrodotoxin was shown in late stage in vitro differentiated human iPSC-CMs [32], thus warranting some caution and further analyses.

Bioanalytics is a very promising tool in the application of in vitro cardiotoxicty assays. Novel bioanalytical tools for discovery of biomarkers of cardiotoxicity include the field potential QT scanning, using cellular oxygen uptake for monitoring the metabolic state of CMs, using surface plasmon resonance (SPR) biosensing for key CM biomarkers, and also exploiting realtime multi-wavelength fluorimetry [12]. Novel imaging technologies and physiological analyses such as impedance measurements [43] and microelectrode arrays (MEAs) [44] give an insight into major in vitro cellular events such as migration, proliferation, cell morphology, cell-cell interactions and colony formation, relevant to biomarker discovery.

\section{Stem cell-derived hepatocytes for toxicity testing}

So far, hepatotoxicity is evaluated on day 28 or 90 in in vivo RDT tests by analysis of clinical parameters, hematology, and histopathology. RDT tests evaluate chronic effects on organ toxicity to establish a NOAEL which is used in calculation of the substance safety parameters [25]. The extrapolation of the quantitative risk assessment for cosmetic ingredients using data derived from animal studies to in vitro systems could be done by considering a margin of safety (MoS) value of at least 100 for intra-species and inter-species variation [25]. Human PSCs represent a promising human cellular model which could help in increasing the safety and predictability of RDT testing. Combined with this cell model, toxicogenomic technologies would help predict biomarkers in an evidence-based approach. During these RDT tests, the animals are observed for indications of toxicity. Afterwards, necropsy, blood analysis and histopathology of the organs of the animals are performed [17]. However, these parameters can turn out to be insensitive and potentially generate false negative results $[15,16]$. Unexpected hepatotoxicity may be seen in the clinical trials or even when the product is already on the market because careful examinations of idiosyncratic (person specific) or non-idiosyncratic inter-drug interactions are either ignored or overseen [45]. This is also probably because of dose-dependent reactions and other unknown peculiar drug interactions. There is need for novel screening methods that can address these hepato-toxicological hazards early in the development [46]. Most studies relied on the use of liver slices as an in vitro model for toxicity testing due to limited availability of tissue samples. However, the human PSC-derived hepatocytes have the potential to replace these in vitro models and be applied for toxicity 
assessment. The suitability of hepatocyte-like cells derived from human PSCs for toxicity testing and drug discovery were systematically studied by several groups [47-49].

\section{Omics strategies to develop biomarkers for RDT}

\subsection{Toxicogenomics}

Proteomics, genomics and metabonomics, either alone or in combination have the potential for developing biomarkers in applied toxicology. Toxicogenomics refers to the areas mentioned above and is a thorough-mean for hi-throughput discovery of biomarkers using latest technologies [50]. Transcriptomics measures the levels of both coding and non-coding RNAs using hi-throughput technology such as microarrays. This whole genome gene expression analysis can measure the levels of expression of a gene at any stage, in any tissue and in any vitro model. Examples of toxicogenomics applications include prediction of genotoxicity or carcinogenicity, target organ toxicity and endocrine disruption. Expression profiling of any selected cellular systems exposed to new test substances is compared against controls to identify, classify and validate toxic compound and its effects. Bioinformatic analyses of the data sets obtained from above can be used to predict the patterns and signatures of a toxin (e.g. biological processes or signaling pathways affected by a toxin). Furthermore, the data sets can be matched up against existing databases for predicting and carving out a mode-of-action for the toxin. The main disadvantage of this approach is limited reproducibility and also it is semi quantitative and detects only changes in gene expression. Therefore, mRNA expression profiling cannot be used as a standalone method in identifying potential biomarkers of RDT.

EU FP7 project Predict-IV is evaluating the integration of 'omics' technologies, biomarkers and high content imaging for the early prediction of toxicity of pharmaceuticals in vitro. The aim is to identify general molecular response pathways that result from toxic drug effects that are independent of the cell/tissue type [51]. Detection of endpoints and biomarkers of RDT using in vitro systems (DETECTIVE) is a unique large scale SEURAT-1 cluster project aimed at establishing screening pipeline of high content, high throughput as well as classical functional and "-omics" technologies to detect human biomarkers for RDT in in vitro test system (http:// www.detect-iv-e.eu/). Other-omics technologies such as microRNA analysis and epigenetics also play a vital role.

\subsection{Proteomics}

Drug induced toxicity can also exhibit various effects at the proteome level. Classification of such endpoints is difficult using traditional RDT methods. Proteomics improves the classification by identifying individual proteins or such protein panels that reflect the specific toxic pathway mechanisms. Proteomics-based in vitro toxicity assays measure drug-induced changes by comparing in vitro to in vivo effects thus validating the suitability of in vitro models. There is an absolute need for integration of standard RDT tests with the 'omics' applications. Current proteomic technologies include gel-based (1-DE or 2-DE) and gel-free (LC-MS/MS) techniques [17]. Recently thalidomide-specific proteomics signatures during 
human ESCs differentiation were identified using two-dimensional electrophoresis coupled with Tandem Mass spectrometry [52]. Proteomic studies are quantitative, sensitive and are more accurate and powerful in detecting protein biomarkers of RDT. Main pitfalls include posttranslational changes and limited protein detection capacity.

\subsection{Metabonomics}

Human PSCs offer a potential alternative test system for the identification of developmental toxicants [53]. Metabonomics refers to profiling of diverse metabolic complement of a biofluid or tissue using analytical tools such as high-field NMR together with mass spectrometry [54]. Subsequent statistical modeling and analysis of a multivariate spectral profiles obtained using NMR [55] in combination with LC-MS and UPLC helps to distinguish the phenotypes and metabolites of interest. These metabolites might represent new biomarkers for toxicity. Previously, some of metabolites were identified to be biomarkers for a variety of pathological diseases [56]. Metabonomics-based approaches have proved to be highly successful in furthering our understanding of research in the field of drug metabolism, drug pathways and toxicology [54,55]. In addition, metabonomics provides a useful link between 'omics' platforms such as genomics, transcriptomics and proteomics and end-stage histopathological analyses [54].

The Consortium for Metabonomic Toxicology (COMET) project (a collaboration between five pharmaceutical companies and Imperial College London) focused on pre-clinical toxicological research and resulted in the generation of an extensive 1H NMR biofluid spectral database which was used for screening of toxins and also to build an expert system for prediction of target organ toxicity [57]. A follow-up project, COMET-2, is currently investigating the detailed biochemical mechanisms of toxicity, and seeks a better understanding of inter-subject variation in metabonomics analyses [55]. Several groups have developed Metabonomics-based robust human ESC in vitro test systems for predicting human developmental toxicity biomarkers and pathways [58,59]. Metabonomics is the most relevant and robust omics platform to study both in vivo and in vitro toxicology. It is possible to detect metabolites with accuracy but it is limited by its high costs and complex metabolite isolation procedures.

\section{Bioinformatic and statistical analysis of candidate biomarkers}

Since the omics methods are extensively data-intensive and bulk, there is a definite need for bioinformatics and statistical analysis for organizing the data in conveniently accessible databases, which integrate huge number of data sets, and therefore need quality database manager software such as SQL for centralized storage and flexible web based access to the bulk data.

Noncommercial databases available on the web such as CEBS (chemical effects in biological systems, (http://cebs.niehs.nih.gov), PhenoGen (http://phenogen.uchsc.edu), along with and commercial databases like ArrayTrack and ArrayExpress (http://www.ebi.ac.uk/arrayexpress) help to generate large data sets. These are complemented by metabonomics databases (http:// 
www.hmdb.ca). PrestOMIC is proteome-specific open-source that is a user-friendly database, where researchers can upload and share data with the scientific community using a customizable browser [60]. Such a database helps researchers to increase the exposure and impact of their data by enabling extensive data set comparisons.

Another open-source systems biology application called SysBio-OM, integrates information from the CEBS database with other open source projects, including MAGE-OM (micro-array gene expression object model) and PEDRo (proteomics experiment data repository), to model profiling of protein, and metabolite expression and protein-protein interactions following insult [61]. SysTox-OM is a more specific application that performs expression profiling of genome, proteome, and metabolome, after the introduction of a toxicant. Different omics approaches and some of the crucial data bases are summarized in Table.1. While incorporating toxicological endpoints such as - clinical chemistry, hematology, observations and histopathology, it profiles the phenotype [62]. With this application, one can identify a single toxic phenotype, classify, and compare gene and protein expression profiles in an organ after administration of each drug. It is also possible to predict a common toxicologic pathway, mechanism or a biomarker. ToxBank is an EU FP7 project aimed at establishing a dedicated web-based warehouse for toxicity data management and modeling along with establishing a cell and tissue banking information for in vitro toxicity testing.

\begin{tabular}{|c|c|c|}
\hline & Databases & Web links \\
\hline \multirow{8}{*}{ 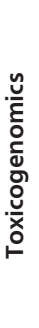 } & Comparative Toxicogenomics Database (CTD) & http://ctdbase.org/ \\
\hline & Open TG-GATEs & http://toxico.nibio.go.jp/english/ \\
\hline & DrugMatrix ${ }^{\circledast}$ & https://ntp.niehs.nih.gov/drugmatrix/index.html \\
\hline & TOXNET & http://toxnet.nlm.nih.gov/index.html \\
\hline & ArrayExpress & http://www.ebi.ac.uk/arrayexpress/ \\
\hline & diXa Data Warehouse & http://wwwdev.ebi.ac.uk/fg/dixa/index.html \\
\hline & ArrayTrack ${ }^{\mathrm{TM}}$ & http://www.fda.gov/ScienceResearch/ \\
\hline & & BioinformaticsTools/Arraytrack/default.htm \\
\hline \multirow{4}{*}{$\begin{array}{l}\frac{\tilde{U}}{\varepsilon} \\
\frac{0}{0} \\
\stackrel{d}{0} \\
\frac{0}{0}\end{array}$} & PRIDE & http://www.ebi.ac.uk/pride/archive/ \\
\hline & ProteomicsDB & https://www.proteomicsdb.org/ \\
\hline & GPMDB & http://gpmdb.thegpm.org/ \\
\hline & NIST & http://peptide.nist.gov/ \\
\hline \multirow{8}{*}{ 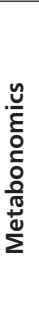 } & Human Metabolome Database (HMDB) & http://www.hmdb.ca/ \\
\hline & BiGG & http://bigg.ucsd.edu/ \\
\hline & MetaboLights & http://www.ebi.ac.uk/metabolights/index \\
\hline & MMCD & http://fiehnlab.ucdavis.edu/projects/binbase_setupx \\
\hline & SetupX \& BinBase & http://www.bml-nmr.org/ \\
\hline & BML-NMR & http://www.massbank.jp/ \\
\hline & MassBank & http://gmd.mpimp-golm.mpg.de/ \\
\hline & Golm Metabolome Database (GMD) & \\
\hline
\end{tabular}

Table 1. Summary of different Omics approaches and corresponding databases 


\section{Conclusion and future perspective}

Improved toxicity testing methods complementing advanced in vitro assays are very crucial in reducing the rate of attrition in final stages of product development. To avoid failures and withdrawals, there is an absolute need for integration of all available technologies to minimize cumbersome process of trials and expenses and eventually reduce the increasing costs of bringing a new drug into market. Supplementing toxicology evaluation methods, such as histopathology, physiology and clinical chemistry with transcriptomics, proteomics and metabonomics could provide new insights into the mechanisms underlying toxicological pathologies. Integration of in vitro toxicology technologies, with systems biology methods resulted in 'systems toxicology'. Expansion of open source databases and analytical platforms is critical to the discovery of novel biomarkers of toxicity. So far, the available approaches for discovery of biomarkers included toxicogenomics, toxicoproteomics, metabonomics and bioinformatics analyses (systems biology approach) while the technologies available for quantification include ELISA, solid phase ELISA, Luminex technology and patterned paper technology [50]. Individual technologies have limited usefulness unless the data generated from these assay platforms and '-omics' discovery technologies are integrated. The discovery of DNA microarrays and protein chips has made information exchanges extraordinarily easy, convenient and quick. Integration of information from these powerful sources using analytical computing software products, noncommercial databases, and advances in hi-throughput technology is the future of the next phase in the identification, selection and qualification of novel biomarkers of toxicity.

Future of toxicogenomics lies in developing a more refined understanding of molecular mechanisms related to specific toxicologies, to elucidate molecular signatures associated with the prediction of biomarkers or panels of biomarkers with support from the field of transcriptomics, metabolomics, and proteomics. These analytical tools applied to the emerging human PSC-based in vitro platforms utilizing their organ-specific differentiated derivatives, such as $\mathrm{CMs}$, hepatocytes and neurons, have a great potential to revolutionize the field of toxicology. However, the full potential of these human in vitro cell-based platforms in predicting toxicity of compounds in humans will be realized only with further improvements in derivation of highly standardized, well-defined and homogeneous cell populations that functionally and structurally strongly resemble their adult counterparts and development of sensitive and robust methods for accurate detection of toxicity.

\section{Acknowledgements}

The work in the author's laboratories is supported by the European Union FP7 Program, Bundesministerium für Bildung und Forschung (BMBF), Else-Kröner-Fresenius Stiftung, Excellence Research Support Program of the University of Cologne and Köln Fortune Program. 


\section{Author details}

Shiva Prasad Potta ${ }^{2}$, Tomo Šarić ${ }^{1}$, Michael Heke ${ }^{1}$, Harinath Bahudhanapati ${ }^{2}$ and Jürgen Hescheler ${ }^{*}$

*Address all correspondence to: J.Hescheler@uni-koeln.de

1 Center for Physiology and Pathophysiology, Institute for Neurophysiology, University of Cologne, Cologne, Germany

2 Tulip Bio-Med Solutions (P) Ltd., Hyderabad, Andhra Pradesh, India

\section{References}

[1] Kola I, Landis J. Can the pharmaceutical industry reduce attrition rates? Nature reviews Drug discovery. 2004;3(8):711-5.

[2] Chapman KL, Holzgrefe H, Black LE, Brown M, Chellman G, Copeman C, et al. Pharmaceutical toxicology: designing studies to reduce animal use, while maximizing human translation. Regulatory toxicology and pharmacology : RTP. 2013;66(1): 88-103.

[3] Hutchinson L, Kirk R. High drug attrition rates--where are we going wrong? Nature reviews Clinical oncology. 2011;8(4):189-90.

[4] Force T, Kolaja KL. Cardiotoxicity of kinase inhibitors: the prediction and translation of preclinical models to clinical outcomes. Nature reviews Drug discovery. 2011;10(2):111-26.

[5] Kannankeril PJ, Roden DM. Drug-induced long QT and torsade de pointes: recent advances. Current opinion in cardiology. 2007;22(1):39-43.

[6] Mordwinkin NM, Burridge PW, Wu JC. A review of human pluripotent stem cell-derived cardiomyocytes for high-throughput drug discovery, cardiotoxicity screening, and publication standards. Journal of cardiovascular translational research. 2013;6(1): 22-30.

[7] Collins BC, Sposny A, McCarthy D, Brandenburg A, Woodbury R, Pennington SR, et al. Use of SELDI MS to discover and identify potential biomarkers of toxicity in InnoMed PredTox: a multi-site, multi-compound study. Proteomics. 2010;10(8): 1592-608.

[8] Kaese S, Verheule S. Cardiac electrophysiology in mice: a matter of size. Frontiers in physiology. 2012;3:345. 
[9] Roden DM, Altman RB, Benowitz NL, Flockhart DA, Giacomini KM, Johnson JA, et al. Pharmacogenomics: challenges and opportunities. Annals of internal medicine. 2006;145(10):749-57.

[10] McKim JM, Jr. Building a tiered approach to in vitro predictive toxicity screening: a focus on assays with in vivo relevance. Combinatorial chemistry \& high throughput screening. 2010;13(2):188-206.

[11] Pollard CE, Valentin JP, Hammond TG. Strategies to reduce the risk of drug-induced QT interval prol.ongation: a pharmaceutical company perspective. British journal of pharmacology. 2008;154(7):1538-43.

[12] Mandenius CF, Steel D, Noor F, Meyer T, Heinzle E, Asp J, et al. Cardiotoxicity testing using pluripotent stem cell-derived human cardiomyocytes and state-of-the-art bioanalytics: a review. Journal of applied toxicology : JAT. 2011;31(3):191-205.

[13] Takahashi K, Tanabe K, Ohnuki M, Narita M, Ichisaka T, Tomoda K, et al. Induction of pluripotent stem cells from adult human fibroblasts by defined factors. Cell. 2007;131(5):861-72.

[14] Adler S, Basketter D, Creton S, Pelkonen O, van Benthem J, Zuang V, et al. Alternative (non-animal) methods for cosmetics testing: current status and future prospects-2010. Archives of toxicology. 2011;85(5):367-485.

[15] Olson H, Betton G, Stritar J, Robinson D. The predictivity of the toxicity of pharmaceuticals in humans from animal data--an interim assessment. Toxicology letters. 1998;102-103:535-8.

[16] Suter L, Schroeder S, Meyer K, Gautier JC, Amberg A, Wendt M, et al. EU framework 6 project: predictive toxicology (PredTox)--overview and outcome. Toxicology and applied pharmacology. 2011;252(2):73-84.

[17] Van Summeren A, Renes J, van Delft JH, Kleinjans JC, Mariman EC. Proteomics in the search for mechanisms and biomarkers of drug-induced hepatotoxicity. Toxicology in vitro : an international journal published in association with BIBRA. 2012;26(3): 373-85.

[18] Bar-Nur O, Russ HA, Efrat S, Benvenisty N. Epigenetic memory and preferential lineage-specific differentiation in induced pluripotent stem cells derived from human pancreatic islet beta cells. Cell stem cell. 2011;9(1):17-23.

[19] Szyf M. Epigenetics, DNA methylation, and chromatin modifying drugs. Annual review of pharmacology and toxicology. 2009;49:243-63.

[20] Liang G, Zhang Y. Genetic and epigenetic variations in iPSCs: potential causes and implications for application. Cell stem cell. 2013;13(2):149-59.

[21] Kim K, Doi A, Wen B, Ng K, Zhao R, Cahan P, et al. Epigenetic memory in induced pluripotent stem cells. Nature. 2010;467(7313):285-90. 
[22] Gupta MK, Illich DJ, Gaarz A, Matzkies M, Nguemo F, Pfannkuche K, et al. Global transcriptional profiles of beating clusters derived from human induced pluripotent stem cells and embryonic stem cells are highly similar. BMC developmental biology. 2010;10:98.

[23] Wilson KD, Venkatasubrahmanyam S, Jia F, Sun N, Butte AJ, Wu JC. MicroRNA profiling of human-induced pluripotent stem cells. Stem cells and development. 2009;18(5):749-58.

[24] Polo JM, Liu S, Figueroa ME, Kulalert W, Eminli S, Tan KY, et al. Cell type of origin influences the molecular and functional properties of mouse induced pluripotent stem cells. Nature biotechnology. 2010;28(8):848-55.

[25] Vanhaecke T, Pauwels M, Vinken M, Ceelen L, Rogiers V. Towards an integrated in vitro strategy for repeated dose toxicity testing. Archives of toxicology. 2011;85(5): 365-6.

[26] Peng S, Lacerda AE, Kirsch GE, Brown AM, Bruening-Wright A. The action potential and comparative pharmacology of stem cell-derived human cardiomyocytes. Journal of pharmacological and toxicological methods. 2010;61(3):277-86.

[27] Jonsson MK, Duker G, Tropp C, Andersson B, Sartipy P, Vos MA, et al. Quantified proarrhythmic potential of selected human embryonic stem cell-derived cardiomyocytes. Stem cell research. 2010;4(3):189-200.

[28] Guo L, Abrams RM, Babiarz JE, Cohen JD, Kameoka S, Sanders MJ, et al. Estimating the risk of drug-induced proarrhythmia using human induced pluripotent stem cellderived cardiomyocytes. Toxicological sciences : an official journal of the Society of Toxicology. 2011;123(1):281-9.

[29] Liang P, Lan F, Lee AS, Gong T, Sanchez-Freire V, Wang Y, et al. Drug screening using a library of human induced pluripotent stem cell-derived cardiomyocytes reveals disease-specific patterns of cardiotoxicity. Circulation. 2013;127(16):1677-91.

[30] Misner DL, Frantz C, Guo L, Gralinski MR, Senese PB, Ly J, et al. Investigation of mechanism of drug-induced cardiac injury and torsades de pointes in cynomolgus monkeys. British journal of pharmacology. 2012;165(8):2771-86.

[31] Brana I, Tabernero J. Cardiotoxicity. Annals of oncology : official journal of the European Society for Medical Oncology / ESMO. 2010;21 Suppl 7:vii173-9.

[32] Sheng X, Reppel M, Nguemo F, Mohammad FI, Kuzmenkin A, Hescheler J, et al. Human pluripotent stem cell-derived cardiomyocytes: response to TTX and lidocain reveals strong cell to cell variability. PloS one. 2012;7(9):e45963.

[33] Sirenko O, Crittenden C, Callamaras N, Hesley J, Chen YW, Funes C, et al. Multiparameter in vitro assessment of compound effects on cardiomyocyte physiology using iPSC cells. Journal of biomolecular screening. 2013;18(1):39-53. 
[34] Harris K, Aylott M, Cui Y, Louttit JB, McMahon NC, Sridhar A. Comparison of electrophysiological data from human-induced pluripotent stem cell-derived cardiomyocytes to functional preclinical safety assays. Toxicological sciences : an official journal of the Society of Toxicology. 2013;134(2):412-26.

[35] Yang X, Pabon L, Murry CE. Engineering adolescence: maturation of human pluripotent stem cell-derived cardiomyocytes. Circulation research. 2014;114(3):511-23.

[36] Zhang Q, Jiang J, Han P, Yuan Q, Zhang J, Zhang X, et al. Direct differentiation of atrial and ventricular myocytes from human embryonic stem cells by alternating retinoid signals. Cell research. 2011;21(4):579-87.

[37] Turnbull IC, Karakikes I, Serrao GW, Backeris P, Lee JJ, Xie C, et al. Advancing functional engineered cardiac tissues toward a preclinical model of human myocardium. FASEB journal : official publication of the Federation of American Societies for Experimental Biology. 2014;28(2):644-54.

[38] Lundy SD, Zhu WZ, Regnier M, Laflamme MA. Structural and functional maturation of cardiomyocytes derived from human pluripotent stem cells. Stem cells and development. 2013;22(14):1991-2002.

[39] Dick E, Rajamohan D, Ronksley J, Denning C. Evaluating the utility of cardiomyocytes from human pluripotent stem cells for drug screening. Biochemical Society transactions. 2010;38(4):1037-45.

[40] Braam SR, Tertoolen L, van de Stolpe A, Meyer T, Passier R, Mummery CL. Prediction of drug-induced cardiotoxicity using human embryonic stem cell-derived cardiomyocytes. Stem cell research. 2010;4(2):107-16.

[41] Yokoo N, Baba S, Kaichi S, Niwa A, Mima T, Doi H, et al. The effects of cardioactive drugs on cardiomyocytes derived from human induced pluripotent stem cells. Biochemical and biophysical research communications. 2009;387(3):482-8.

[42] Binah O, Weissman A, Itskovitz-Eldor J, Rosen MR. Integrating beat rate variability: from single cells to hearts. Heart rhythm : the official journal of the Heart Rhythm Society. 2013;10(6):928-32.

[43] Nguemo F, Saric T, Pfannkuche K, Watzele M, Reppel M, Hescheler J. In vitro model for assessing arrhythmogenic properties of drugs based on high-resolution impedance measurements. Cellular physiology and biochemistry : international journal of experimental cellular physiology, biochemistry, and pharmacology. 2012;29(5-6): 819-32.

[44] Reppel M, Boettinger C, Hescheler J. Beta-adrenergic and muscarinic modulation of human embryonic stem cell-derived cardiomyocytes. Cellular physiology and biochemistry : international journal of experimental cellular physiology, biochemistry, and pharmacology. 2004;14(4-6):187-96.

[45] Hartung T. Toxicology for the twenty-first century. Nature. 2009;460(7252):208-12. 
[46] Amacher DE. The discovery and development of proteomic safety biomarkers for the detection of drug-induced liver toxicity. Toxicology and applied pharmacology. 2010;245(1):134-42.

[47] Takayama K, Kawabata K, Nagamoto Y, Kishimoto K, Tashiro K, Sakurai F, et al. 3D spheroid culture of hESC/hiPSC-derived hepatocyte-like cells for drug toxicity testing. Biomaterials. 2013;34(7):1781-9.

[48] Ulvestad M, Nordell P, Asplund A, Rehnstrom M, Jacobsson S, Holmgren G, et al. Drug metabolizing enzyme and transporter protein profiles of hepatocytes derived from human embryonic and induced pluripotent stem cells. Biochemical pharmacology. 2013;86(5):691-702.

[49] Yildirimman R, Brolen G, Vilardell M, Eriksson G, Synnergren J, Gmuender H, et al. Human embryonic stem cell derived hepatocyte-like cells as a tool for in vitro hazard assessment of chemical carcinogenicity. Toxicological sciences : an official journal of the Society of Toxicology. 2011;124(2):278-90.

[50] Collings FB, Vaidya VS. Novel technologies for the discovery and quantitation of biomarkers of toxicity. Toxicology. 2008;245(3):167-74.

[51] Adler S, Lindqvist J, Uddenberg K, Hyllner J, Strehl R. Testing potential developmental toxicants with a cytotoxicity assay based on human embryonic stem cells. Alternatives to laboratory animals : ATLA. 2008;36(2):129-40.

[52] Meganathan K, Jagtap S, Wagh V, Winkler J, Gaspar JA, Hildebrand D, et al. Identification of thalidomide-specific transcriptomics and proteomics signatures during differentiation of human embryonic stem cells. PloS one. 2012;7(8):e44228.

[53] Chapin RE, Stedman DB. Endless possibilities: stem cells and the vision for toxicology testing in the 21st century. Toxicological sciences : an official journal of the Society of Toxicology. 2009;112(1):17-22.

[54] Coen M. A metabonomic approach for mechanistic exploration of pre-clinical toxicology. Toxicology. 2010;278(3):326-40.

[55] Nicholson JK, Lindon JC. Systems biology: Metabonomics. Nature. 2008;455(7216): 1054-6.

[56] Holmes E, Wilson ID, Nicholson JK. Metabolic phenotyping in health and disease. Cell. 2008;134(5):714-7.

[57] Ebbels TM, Keun HC, Beckonert OP, Bollard ME, Lindon JC, Holmes E, et al. Prediction and classification of drug toxicity using probabilistic modeling of temporal metabolic data: the consortium on metabonomic toxicology screening approach. Journal of proteome research. 2007;6(11):4407-22. 
[58] West PR, Weir AM, Smith AM, Donley EL, Cezar GG. Predicting human developmental toxicity of pharmaceuticals using human embryonic stem cells and metabolomics. Toxicology and applied pharmacology. 2010;247(1):18-27.

[59] Kleinstreuer NC, Smith AM, West PR, Conard KR, Fontaine BR, Weir-Hauptman $\mathrm{AM}$, et al. Identifying developmental toxicity pathways for a subset of ToxCast chemicals using human embryonic stem cells and metabolomics. Toxicology and applied pharmacology. 2011;257(1):111-21.

[60] Howes CG, Foster LJ. PrestOMIC, an open source application for dissemination of proteomic datasets by individual laboratories. Proteome science. 2007;5:8.

[61] Xirasagar S, Gustafson S, Merrick BA, Tomer KB, Stasiewicz S, Chan DD, et al. CEBS object model for systems biology data, SysBio-OM. Bioinformatics. 2004;20(13): 2004-15.

[62] Xirasagar S, Gustafson SF, Huang CC, Pan Q, Fostel J, Boyer P, et al. Chemical effects in biological systems (CEBS) object model for toxicology data, SysTox-OM: design and application. Bioinformatics. 2006;22(7):874-82. 